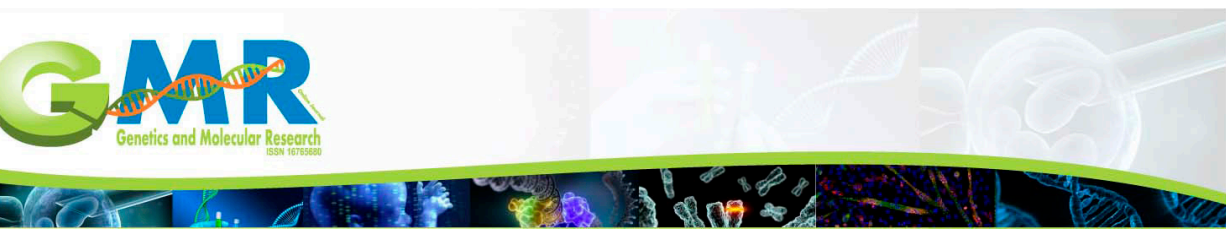

\title{
Absence of the BaeR protein leads to the early initiation of DNA replication in Escherichia coli
}

\author{
Y. Yao, Wunier and Morigen \\ School of Life Sciences, Inner Mongolia University, Hohhot, China \\ Corresponding author: Morigen \\ E-mail: morigenm@hotmail.com
}

Genet. Mol. Res. 14 (4): 16888-16895 (2015)

Received June 30, 2015

Accepted September 11, 2015

Published December 14, 2015

DOI http://dx.doi.org/10.4238/2015.December.14.16

ABSTRACT. Escherichia coli cells have dozens of two-component systems to sense and respond to various stimuli, and thereby cope with changing environments. BaeS/BaeR is one such two-component system, and it deals with a variety of envelope stresses. Interestingly, the ArcA/ArcB and TorS/ TorR two-component systems are known to be associated with initiation of DNA replication; however, the effects of BaeS/BaeR on initiation of DNA replication remain unknown. Flow cytometry analysis revealed that the average number of replication origins (oriCs) per cell in $\Delta$ bae $R$ mutants was approximately $30 \%$ higher than that in wild-type cells. So was the growth rate of $\triangle b a e R$ cells. Ectopic expression of BaeR from the pbae $R$ plasmid reversed the $\triangle b a e R$ mutant phenotypes. The results indicate that absence of BaeR leads to the early initiation of DNA replication. Further, deletion of BaeR caused an increase in the amount of DnaA per cell, but did not change concentration of DnaA, which is the initiator protein. The average number of oriCs per cell in $\Delta s p y$ mutants was the same as that found in the wild-type cells although spy gene expression is controlled by BaeR. These results suggest that BaeR may indirectly affect initiation of replication by controlling expression of the $d n a A$ gene.

Key words: Response regulator BaeR; DNA replication; Escherichia coli 


\section{INTRODUCTION}

Bacterial cells have dozens of two-component systems (TCS) that sense and respond to specific stimuli and subsequently allow the cells to cope with a variety of environmental conditions. A classical TCS includes a histidine kinase (HK) on the membrane and a response regulator in the cytoplasm. HK can be phosphorylated because of environmental stimuli, and the phosphate group can be transferred to its response regulator. The Escherichia coli envelope (inner membrane, periplasm, outer membrane, and bacterial appendages) is exposed to a variety of environmental stresses, including changes in temperature, $\mathrm{pH}$, and osmolarity, toxic compounds, and oxidative stress (Leblanc et al., 2011). E. coli cells possess different kinds of stress signaling systems, one of which, BaeS/BaeR, senses and defends against divalent ion toxicity (Ruiz and Silhavy, 2005). BaeS/BaeR is a TCS consisting of a sensor HK, BaeS, and a response regulator (RR), BaeR (Raffa and Raivio, 2002). When BaeS senses changes in the concentration of divalent ions, a phosphotransfer from the conserved histidine residue of BaeS to the aspartate residue in the cognate response regulator BaeR occurs after a second round of autophosphorylation (Nagasawa, et al., 1993; Raffa and Raivio, 2002). Phosphorylated BaeR as a dimer actively binds to its target promoters and subsequently regulates expression of a number of genes ( $m d t A B C D$, acrD, baeSR, spy, tolC, ygiABC, ynjABCD, and yeeN) (Oshima et al., 2002; Nishino et al., 2005). These genes are associated with cell adaptation to envelope stress.

It has been shown that the $\mathrm{CtrA}, \mathrm{ArcB}, \mathrm{PhoQ}$, and TorR RRs are associated with the initiation of DNA replication (Soncini and Groisman, 1996; Colloms et al., 1998; Jacobs et al., 2003; Yuan et al., 2015). Here we show that the absence of BaeR leads to the early initiation of DNA replication.

\section{MATERIAL AND METHODS}

\section{Bacterial strains and plasmids}

The E. coli K-12 bacterial strains and the plasmids used in this study are listed in Table 1. The baeR gene was amplified by polymerase chain reaction (PCR), using the chromosomal DNA of strain BW25113 (Baba et al., 2006) as a template and the primers 5'-CGGGATCCGAGAGAAG TATGACCGAGTTAC-3' and 5'-CCCAAGCTTCTAAACGATGCGGCAGGCG-3'. The resultant fragment was inserted at the BamHI and HindIII sites in pUHE21, where the expression of baeR is under the control of the lac promoter (Soncini et al., 1995), leading to the construction of the pbaeR plasmid. The plasmid was introduced into competent cells by $\mathrm{CaCl}_{2}$ transformation.

\begin{tabular}{|c|c|c|}
\hline Strains and plasmids & Relevant genotype & Source or reference \\
\hline BW25113 & $\begin{array}{l}\text { Wild-type } r r n B 3 \Delta / a c Z 4787 \text { hsdR514 } \\
\Delta(\text { araBAD }) 567 \Delta(\text { rhaBAD }) 568 \text { rph-1 }\end{array}$ & (Baba et al., 2006) \\
\hline MOR393 & BW25113 spy::kan & (Baba et al., 2006) \\
\hline MOR452 & BW25113 baeR::kan & (Baba et al., 2006) \\
\hline MOR1195 & BW25113/pbaeR & This study \\
\hline MOR1196 & BW25113/pUHE21-2lac ${ }^{q}$ & This study \\
\hline MOR1199 & MOR452/pbaeR & This study \\
\hline MOR1209 & MOR452/pUHE21-2/ac ${ }^{q}$ & This study \\
\hline pUHE21-2lac q & $\operatorname{rep}_{\mathrm{pMB} 1} \mathrm{Ap}^{\mathrm{R}} / \mathrm{ac} /^{\mathrm{q}}$ & (Soncini et al., 1995) \\
\hline pbaeR & baeR gene on pUHE21-2lac & This study \\
\hline
\end{tabular}




\section{Growth media and conditions}

Cells were exponentially grown in ABTGcasa medium (Morigen et al., 2005) at $37^{\circ} \mathrm{C}$. Ampicillin $(50 \mu \mathrm{g} / \mathrm{mL})$, kanamycin $(50 \mu \mathrm{g} / \mathrm{mL})$, and chloramphenicol $(30 \mu \mathrm{g} / \mathrm{mL})$ were added when required for selection. To induce the expression of BaeR from the pbae $R$ plasmid at $37^{\circ} \mathrm{C}, 0.25 \mathrm{mM}$ isopropyl $\beta$-D1-thiogalactopyranoside (IPTG) was added to the exponentially growing cells in ABTGcasa medium.

\section{Flow cytometry}

Exponentially growing cells in ABTGcasa medium at $37^{\circ} \mathrm{C}$ were treated with $300 \mu \mathrm{g} /$ $\mathrm{mL}$ rifampicin and $10 \mu \mathrm{g} / \mathrm{mL}$ cephalexin for 3-4 generations. Rifampicin prevents the initiation of replication by inhibiting transcription, which is required for the initiation of replication but allows the completion of ongoing replication. Cephalexin blocks cell division at the time of addition of the drug (Skarstad et al.,1986; Boye and Løbner-Olesen, 1991). The rifampicin- and cephalexin-treated cells were fixed in $70 \%$ ethanol. The cells were stained with Hoechst 33258 for 30 min after one wash in Tris- $\mathrm{HCl}$ buffer ( $\mathrm{pH} 7.5$ ), and subsequently analyzed using an LSRFortessa flow cytometer (BD Biosciences, San Jose, CA, USA). Preparation of standard samples and post analysis were as described previously (Morigen et al., 2003).

\section{Determination of total protein per cell}

Exponentially growing cells in ABTGcasa at $37^{\circ} \mathrm{C}$ were collected on ice. $9 \mathrm{~mL}$ of the cell culture were harvested by centrifugation at $4^{\circ} \mathrm{C}$, washed in $1 \mathrm{~mL}$ of TE buffer, and resuspended in $200 \mu \mathrm{L}$ TE buffer containing 1\% SDS and glycerol and then boiled for $5 \mathrm{~min}$. Total protein amount in a fixed volume of the cell extract mentioned above was determined by a colorimetric assay (BCA kit, pierce) as described previously (Morigen et al., 2003). The number of cells in a certain volume of the cell culture and the protein amount per cell was dermined as described previously (Liu et al., 2014).

\section{Western blotting}

Determination of the DnaA concentration in the cell extract mentioned above as mentioned previously (Morigen et al., 2001).

\section{RESULTS}

\section{Deletion of the baeR gene leads to the early initiation of DNA replication}

To investigate whether the BaeR response regulator from BaeS/BaeR also affects the initiation of DNA replication, we analyzed the replication patterns of the $\triangle b a e R$ mutant and wildtype cells by flow cytometry. In the wild-type cells 2-, 4- and a small proportion of 8-origin cells were detected, whereas only 4 - and 8-origin cells were found in the $\Delta$ baeR mutant (Figure 1). The average number of origins for replication (oriCs) per cell was 4.4 for the wild-type cells and 5.7 for the $\triangle$ baeR mutant (Figure 1, Table 2), an increase of $30 \%$ in the $\Delta b a e R$ mutant relative to the wildtype cells. Concomitantly, the growth rate increased slightly (the doubling time was 34 min for the wild-type cells and $31 \mathrm{~min}$ for the mutant) (Table 2). The results indicate that the absence of BaeR leads to the early initiation of DNA replication relative to that in the wild-type cells. 


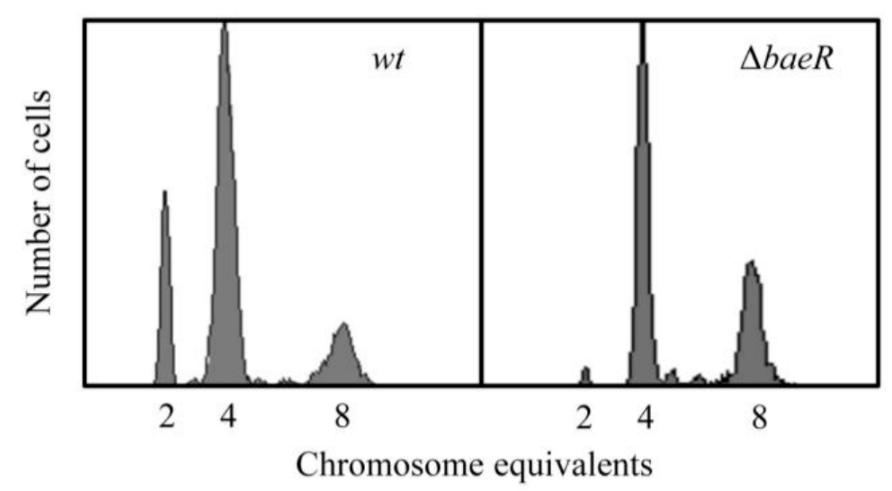

Figure 1. Deletion of the baeR gene results in the early initiation of replication. Exponentially growing cells in ABTGcasa medium at $37^{\circ} \mathrm{C}$ were treated with rifampicin and cephalexin for 3-4 generations. Subsequently, cells were fixed in $70 \%$ ethanol and analyzed by flow cytometry, as described in the Material and Methods section. The number of fully replicated chromosomes per cell represents the number of origins present at the time of drug addition. For each analysis, 10,000 cells were included.

Table 2. Absence of BaeR leads to an increase in the number of origins per cell.

\begin{tabular}{llcc}
\hline Strains & Genotype & A.O. & Doubling time (min) \\
\hline BW25113 & Wild-type & $4.4 \pm 0.1$ & $34 \pm 0.6$ \\
MOR393 & $\Delta s p y$ & $4.3 \pm 0.1$ & $34 \pm 0.4$ \\
MOR452 & $\Delta$ bae $R$ & $5.7 \pm 0.7$ & $31 \pm 1.2$ \\
MOR1195 & BW25113/pbaeR & $4.1 \pm 0.2$ & $38 \pm 0.6$ \\
MOR1196 & BW2513/puHE21-2lac & $4.3 \pm 0.1$ & $35 \pm 0.2$ \\
MOR1199 & $\Delta$ baeR/pbaeR & $4.2 \pm 0.1$ & $37 \pm 0.6$ \\
MOR1209 & $\Delta$ baeR/pUHE21-2lac & $5.4 \pm 0.0$ & $32 \pm 0.1$ \\
\hline
\end{tabular}

Exponentially growing cells in ABTGcasa medium were treated with rifampicin and cephalexin, fixed in $70 \%$ ethanol, and then analyzed by flow cytometry, as described in the Material and Methods section. The average number of origins per cell (A.O.) was calculated using software provided by BD Biosciences. Each experiment was repeated three times and standard errors are given.

\section{Ectopically expressed BaeR reverses the replication pattern of the $\Delta b a e R$ mutant}

To ascertain whether ectopically expressed BaeR can reverse the replication pattern of the $\triangle b a e R$ mutant, we constructed the pbaeR plasmid, which expresses BaeR under the control of the lac promoter. The wild-type and $\triangle b a e R$ mutant cells were transformed with the plasmid pbaeR or the control vector. Exponentially growing cells containing pbaeR or the control vector in ABTGcasa medium with IPTG to induce BaeR expression, were treated with rifampicin and cephalexin, stained with Hoechst, and analyzed by flow cytometry, as mentioned above. The $\Delta b a e R$ mutant expression of BaeR from pbaeR produced cells with 2, 4, or 8 origins and an extended doubling time (37 min compared with $31 \mathrm{~min}$ for cells with the control vector) (Figure 2, Table 2). The average number of origins per cell decreased from 5.7 in the $\triangle b a e R$ cells carrying the control vector to 4.2 in the $\triangle b a e R$ cells containing pbae $R$ (Figure 2, Table 2). When the BaeR protein was ectopically overexpressed in the wild-type cells, the DNA replication pattern was similar to that in the wild-type cells without excess BaeR, containing 2, 4, and 8 origins (Figure 2), and the average number of origins per cell decreased slightly (Table 2). The results indicate that ectopically expressed BaeR reverses the $\triangle b a e R$ mutant phenotype. 


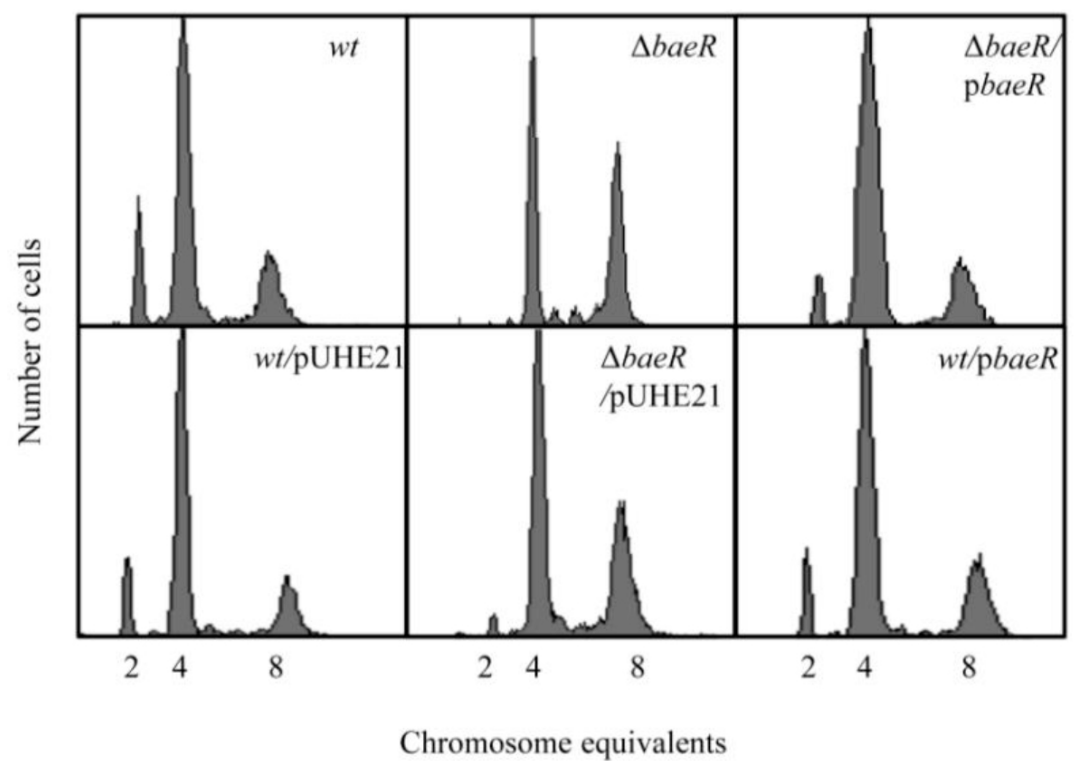

Figure 2. Ectopically expressed BaeR reverses the $\triangle$ baeR mutant phenotype. Exponentially growing cells in ABTGcasa medium containing isopropyl $\beta$-D-1-thiogalactopyranoside (IPTG) $(0.25 \mathrm{mM})$ to induce expression of BaeR from the pbae $R$ plasmid at $37^{\circ} \mathrm{C}$ were treated with rifampicin and cephalexin for 3-4 generations and then analyzed by flow cytometry as described in the Material and Methods section.

\section{BaeR affects the amount of DnaA per cell}

The DnaA protein is the initiatior for DNA replication (Braun et al., 1985; Løbner-Olesen et al., 1989). Therefore, the DnaA protein amount and/or concentration have been known as a limiting factor for initiation of replication. It is likely that BaeR affects initiation of replication by altering the amount or concentration of DnaA. In order to detect this posibility, we measured the amount of DnaA protein per cell. Wide type and $\triangle b a e R$ cells with or without overexpressed BaeR were exponentially grown in $A B T G$ casa medium at $37^{\circ} \mathrm{C}$ and the total amount of protein per cell was measured as described in Material and Methods. Also the DnaA concentration in these cell extracts were measured by Western blotting. The amount of DnaA protein per cell in $\triangle b a e R$ cells was increased relative to that in wide-type (Figure $3 A$ ). In addition, we found that the total amount of protein per cell in $\Delta b a e R$ cells was also observed to be increased compared with that in wide-type (Figure 3B). The results indicate that BaeR affects the initiation by changing the amount of DnaA per cell.

\section{Absence of the spy gene does not affect initiation of DNA replication}

The BaeR protein regulates expression levels of a number of genes including the spy gene. BaeR may indirectly affect the initiation of replication by controlling the expression of its target genes. To test this possibility, we analyzed the replication pattern of the $\Delta s p y$ mutant cells, as described above, since expression of the spy gene is controlled by BaeR. Flow cytometry analysis showed that the $\Delta$ spy mutant cells had the wild-type DNA replication pattern with a doubling time of $34 \mathrm{~min}$ (Figure 4, Table 2). The results suggest that BaeR does not affect initiation of DNA replication by regulating expression of the spy gene. 

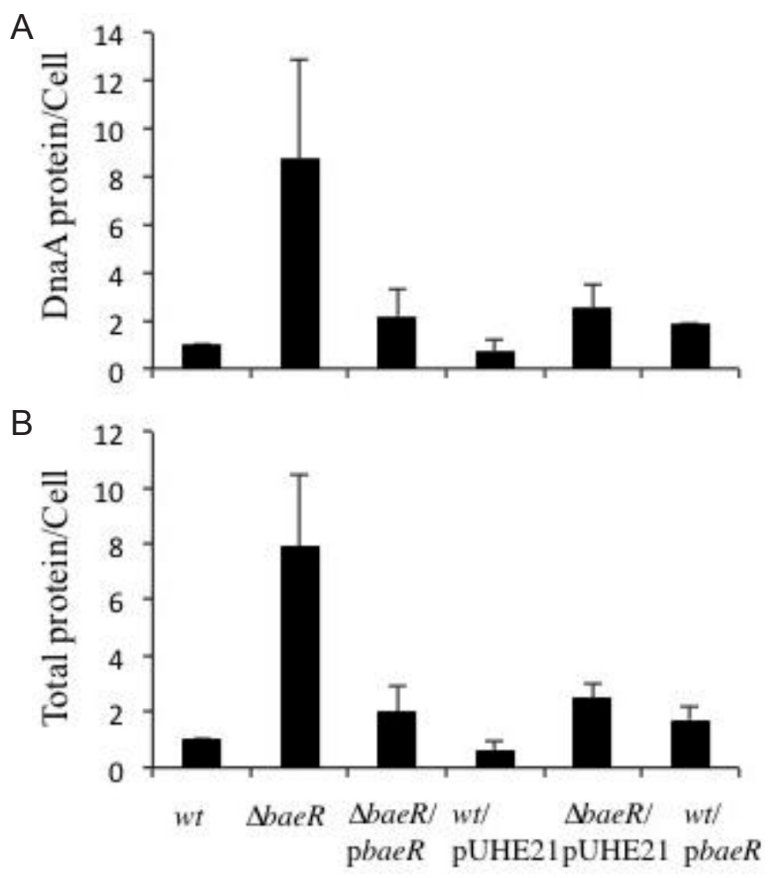

Figure 3. A. BaeR affects the amount of DnaA per cell. A. The amount of DnaA protein per cell is increased in $\triangle b a e R$ cells. Exponentially growing cells in ABTGcasa at $37^{\circ} \mathrm{C}$ were harvested by centrifugation at $4^{\circ} \mathrm{C}$. B. Total protein amount in a fixed volume of cell extract was determined by a colorimetric assay (BCA kit) as described previously (Liu et al., 2014). The DnaA concentration was determined by immunobloting. The DnaA protein amount per cell was then estimated through counting the number of cells used in the measurement.

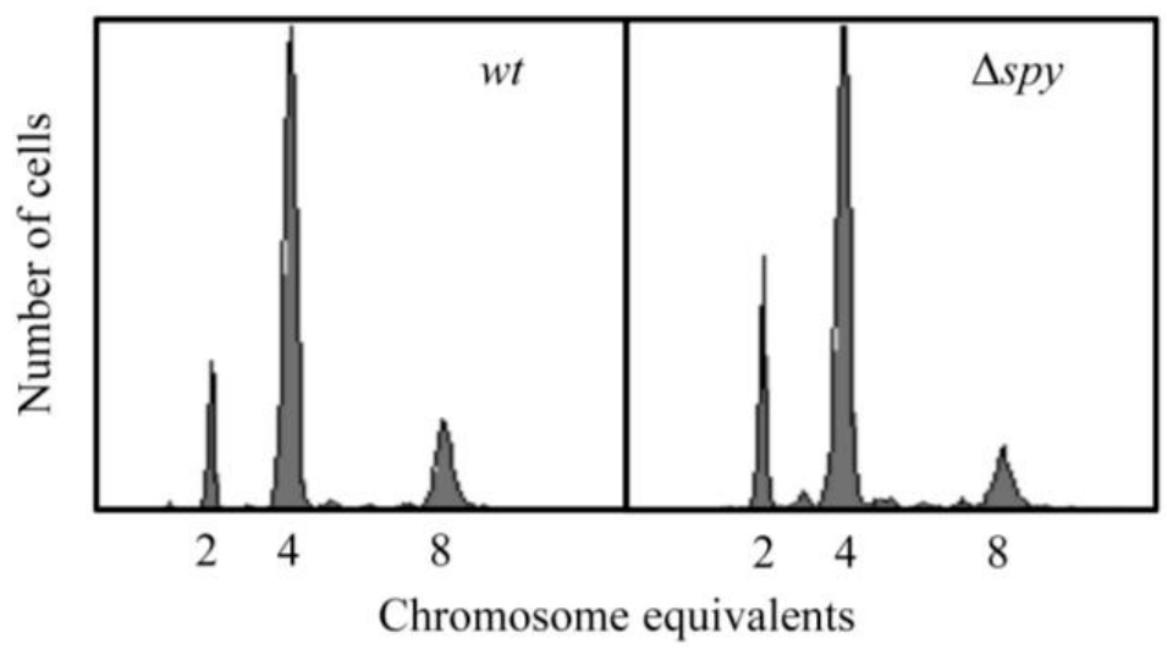

Figure 4. DNA replication pattern is not changed in the $\Delta$ spy mutant. Exponentially growing cells in ABTGcasa medium at $37^{\circ} \mathrm{C}$ were treated with rifampicin and cephalexin for 3-4 generations and then analyzed by flow cytometry as described in the Material and Methods section. 


\section{DISCUSSION}

In this study, we found that the absence of the BaeR protein led to the early initiation of DNA replication. Furthermore, ectopically expressed BaeR restores the wild-type replication pattern in the $\triangle b a e R$ mutant cells. BaeR affects the initiation of DNA replication either directly or indirectly. It is likely that BaeR may indirectly affect the initiation of replication by regulating expression of its target genes such as the spy gene (Oshima et al., 2002; Nishino et al., 2005). However, deletion of the spy gene results in a wild-type replication pattern, suggesting that the Spy protein is not associated with initiation of replication. It could also be possible that the products from $m d t A B C D$, acrD, baes, tolC, ygiABC, ynjABCD, and yeeN may affect the initiation of replication since the expression of these genes is controlled by BaeR (Oshima et al., 2002; Nishino et al., 2005). Alternatively, the expression of the initiator protein DnaA could increase in the absence of BaeR. Indeed, the amount of DnaA protein was increased in $\triangle b a e R$ cells.

Moreover, bacterial cells have dozens of TCS; the E. coli ArcA/ArcB and TorS/TorR TCS are associated with the initiation of DNA replication (Soncini and Groisman, 1996; Colloms et al., 1998; Jacobs et al., 2003; Yuan et al., 2015), although their underlying mechanisms remain elusive.

\section{CONFLICTS OF INTEREST}

The authors declare no conflict of interest.

\section{ACKNOWLEDGMENTS}

Research supported by grants from the National Natural Science Foundation of China "NSFC" (Grant \#31360208 to Morigen); the Natural Science Foundation of Inner Mongolia (Grant \#20102009 to Morigen); and the Program of Higher-Level Talents of Inner Mongolia University "SPH-IMU" (Grant \#Z20090107 to Morigen).

\section{REFERENCES}

Baba T, Ara T, Hasegawa M, Takai Y, et al. (2006). Construction of Escherichia coli K-12 in-frame, single-gene knockout mutants: the Keio collection. Mol. Syst. Biol. 2: 2006.0008.

Boye E and Løbner-Olesen A (1991). Bacterial growth control studied by flow cytometry. Res. Microbiol. 142: 131-135.

Braun RE, O'Day K and Wright A. (1985). Autoregulation of the DNA replication gene dnaA in E. coli K-12. Cell 40: 159-169.

Colloms SD, Alén C and Sherratt DJ (1998). The ArcA/ArcB two-component regulatory system of Escherichia coli is essential for Xer site-specific recombination at psi. Mol. Microbiol. 28: 521-530.

Jacobs C, Ausmees N, Cordwell SJ, Shapiro L, et al. (2003). Functions of the CckA histidine kinase in Caulobacter cell cycle control. Mol. Microbiol. 47: 1279-1290.

Kitagawa M, Ara T, Arifuzzaman M, loka-Nakamichi T, et al. (2006). Complete set of ORF clones of Escherichia coli ASKA library (a complete set of $E$. coli K-12 ORF archive): unique resources for biological research. DNA Res. 12: 291-299.

Leblanc SK, Oates CW and Raivio TL (2011). Characterization of the induction and cellular role of the BaeSR two-component envelope stress response of Escherichia coli. J. Bacteriol. 193: 3367-3375.

Liu F, Qimuge, Hao J, Yan H, et al. (2014). Aspc-mediated aspartate metabolism coordinates the Escherichia coli cell cycle. Plos One 9: e92229.

Løbner-Olesen A, Skarstad K, Hansen FG and von Meyenburg K, et al. (1989). The DnaA protein determines the initiation mass of Escherichia coli K-12. Cell 57: 881-889.

Morigen, Boye E, Skarstad K, Løbner-Olesen A (2001). Regulation of chromosomal replication by DnaA protein availability in Escherichia coli: effects of the datA region. BBA-Gene Struct. Expr. 1521: 73-80.

Morigen, Løbner-Olesen A and Skarstad K (2003). Titration of the Escherichia coli DnaA protein to excess datA sites causes destabilization of replication forks, delayed replication initiation and delayed cell division. Mol. Microbiol. 50: 349-362. 
Morigen, Molina F and Skarstad K (2005). Deletion of the datA site does not affect once-per-cell-cycle timing but induces rifampin-resistant replication. J. Bacteriol. 187: 3913-3920.

Nagasawa S, Ishige K and Mizuno T (1993). Novel members of the two-component signal transduction genes in Escherichia coli. J. Biochem. 114: 350-357.

Nishino K, Honda T and Yamaguchi A (2005). Genome-wide analyses of Escherichia coli gene expression responsive to the BaeSR two-component regulatory system. J. Bacteriol. 187: 1763-1772.

Oshima T, Aiba H, Masuda Y, Kanaya S, et al. (2002). Transcriptome analysis of all two-component regulatory system mutants of Escherichia coli K-12. Mol. Microbiol. 46: 281-291.

Raffa RG and Raivio TL (2002). A third envelope stress signal transduction pathway in Escherichia coli. Mol. Microbiol. 45: 1599-1611.

Ruiz N and Silhavy TJ (2005). Sensing external stress: watchdogs of the Escherichia coli cell envelope. Curr. Opin. Microbiol. 8: $122-126$

Skarstad K, Boye E and Steen HB (1986). Timing of initiation of chromosome replication in individual Escherichia coli cells. EMBO J. 5: 1711-1717.

Soncini FC and Groisman EA (1996). Two-component regulatory systems can interact to process multiple environmental signals. J. Bacteriol. 178: 6796-6801.

Soncini FC, Véscovi EG and Groisman EA (1995). Transcriptional autoregulation of the Salmonella typhimurium phoPQ operon. J. Bacteriol. 177: 4364-4371.

Yuan Y, Jiaxin Q, Jing L, Hui L, et al. (2015). [The effects of TorR protein on initiation of DNA replication in Escherichia coll]. Yi Chuan. 37: 302-308. 